\title{
LA PRODUCCIÓN DEL ESPACIO URBANO Y LA INSTITUCIONALIZACIÓN DE PRÁCTICAS DE ASOCIACIÓN PÚBLICO-PRIVADAS. Convenios urbanísticos en la ciudad de Córdoba: ¿Hacia la corporativización de la producción del suelo urbano?
}

\author{
Autora: Natalí Peresini \\ Institución de pertenencia: CONICET-CEVE - Doctorado en Estudios urbanos-UNGS \\ Director de tesis: Dr. Luis Baer \\ Email: natperesini@gmail.com
}

\section{RESUMEN}

Como manifestación del proceso de urbanización contemporáneo, han surgido en Latinoamérica novedosas formas de producción del espacio urbano, que implican también nuevas relaciones entre la gestión y los agentes que lo producen. La creciente utilización de instrumentos de gestión urbana presenta signos de cambios estructurales en la gestión pública que merecen ser estudiados, nos enfocaremos particularmente en los Acuerdos Público Privados.

Esta ponencia expondrá avances del trabajo doctoral que tiene como objetivo estudiar la institucionalización de prácticas de asociación horizontal entre gobiernos locales y desarrollistas inmobiliarios, tomando por caso la ciudad de Córdoba tras la implementación de los "convenios urbanísticos" (2012); la presentación se centrará en el análisis de los marcos regulatorios y su relación con las dinámicas de desarrollo urbano, prestando especial atención al último período para reconocer la incidencia que tienen los APP en el proceso de urbanización y su relación con la última generación de emprendimientos inmobiliarios.

Palabras clave: Producción del espacio -Instrumentos de gestión urbana - APP -

\begin{abstract}
As a manifestation of the contemporary urbanization process, new forms of urban space production have emerged in Latin America, which also imply new relationships between urban management and the agents that produce it. The growing use of urban management instruments presents signs of structural changes in public management that deserve to be studied, we will focus particularly on Public Private Partnership.

This paper will present advances in doctoral work that aims to study the institutionalization of horizontal association practices between local governments and real estate developers, taking the case of the Córdoba city after the implementation of the "convenios urbanísticos" (2012); this presentation will focus on the analysis of the regulatory frameworks and their relationship with the dynamics of urban development, paying attention to the last period by studying the impact that PPPs have on the urbanization process and its relationship with the latest generation of real estate development.
\end{abstract}

Key words: Production of space -instruments of urban management - Public Private Partnership - 


\section{INTRODUCCIÓN}

En Latinoamérica, el proceso de urbanización en el actual período neoliberal presenta nuevas formas de producción del espacio que implican también novedosas relaciones entre la gestión urbana, las nuevas modalidades de producción y los agentes que la producen (Abramo, 2009). Paralelamente a este proceso, en el último veinteno ha surgido en Latinoamérica un renovado interés por instrumentos específicos para la gestión urbana implementándose a nivel nacional, como lo es la Ley de Ordenamiento Territorial colombiana o el Estatuto da Cidade en Brasil que han motivado numerosas investigaciones sobre las novedosas experiencias. La amplia batería de instrumentos de gestión urbana institucionalizados en marcos jurídicos específicos -y las investigaciones en torno a su empleo e impacto en la ciudad- discrepa con la situación local; para el caso de Argentina cabe distinguir algunos aspectos estructurales que determinan sus prácticas respecto a la de los países mencionados. En primer lugar, la valoración sacrílega de la propiedad privada adoptada en el código civil y que rige en la regulación jurídica y por otro lado, la autonomía municipal de los gobiernos locales para concretar la estructura espacial a través de normas administrativas que regulan los procesos de creación y uso del suelo urbano; por lo que la normativización, aplicación y sistematización de los instrumentos y estrategias no se han registrado sino como prácticas de las administraciones locales y de acuerdo a las posibilidades coyunturales en la lucha de intereses entre los agentes. ${ }^{1}$

Dentro del "catálogo" de instrumentos disponibles en el ejercicio de gestionar las ciudades, nos encontramos con intervenciones específicas relacionadas al financiamiento del desarrollo urbano, la recaudación fiscal o la democratización de la gestión urbana. En nuestro país, llama la atención el particular interés por aquellos que permiten un nuevo rol de las esferas gubernamentales, específicamente la incursión del gobierno local con lógicas de actuación similares a las del mercado. Así pues, la noción de la recuperación de plusvalía y las intervenciones en el mercado de suelo, han sido esgrimidas como las principales argumentaciones y motivaciones para la aplicación de los mecanismos implementados. Enfocándonos particularmente en la tendencia de utilización de Acuerdos Público Privados (APP), la base consiste en combinar el poder del gobierno local en la toma de decisiones, aval, regularización e institucionalización de las prácticas con el capital y la capacidad técnica del privado. Esta estrategia, demuestra la fuerte tendencia privatista en la gestión urbana que más que utilizar un instrumento de gestión-administrativo-normativo novedoso, introduce cambios estructurales en la gestión pública con raíz en dos ejes fundamentales: la concepción de ciudad y lo que ella representa y la lógica de actuación-intervención pública a través de sus políticas urbanas (Vainer, 2002). En este contexto, el uso intensivo de este instrumento y las dinámicas particulares que ha provocado obligan a revisar sus efectos en el proceso de urbanización y las transformaciones sufridas en la organización socioespacial de las ciudades.

El trabajo doctoral abordará como objeto de estudio las vinculaciones público-privadas aplicadas en la gestión urbana, su influencia en el proceso de urbanización y en la dinámica de producción del espacio. Lo que interesa estudiar se apoya en este fenómeno, tomando como caso de estudio la ciudad de Córdoba a partir de la ordenanza de "convenios urbanísticos" (Córdoba, 2012), para conceptualizar y profundizar en el conocimiento de nuevas prácticas de asociación horizontal entre los gobiernos locales y desarrollistas inmobiliarios y las características de las urbanizaciones generadas a partir de esta novedosa modalidad institucionalizada en el marco regulatorio local. Como hipótesis central manifestamos que la asociación de las empresas desarrollistas con los gobiernos locales cristaliza nuevas estrategias empresarialistas que logran direccionar y hegemonizar la producción del suelo urbano, constituyendo una nueva etapa del proceso de urbanización neoliberal. Esta ponencia expondrá los avances de la investigación en curso, puntualmente la evolución de los marcos regulatorios y su relación con las dinámicas y patrones de desarrollo urbano, prestando especial interés al último período estudiando la real incidencia que tienen las APP en el proceso de urbanización y su relación con la última generación de emprendimientos inmobiliarios, con el fin de comprender la influencia de las vinculaciones público-privadas en la producción del espacio. Para ello, se presentará una descripción y análisis de la trayectoria de los marcos regulatorios asociados a las transformaciones espaciales y su relación con las distintas generaciones de emprendimientos inmobiliarios en la ciudad de Córdoba a partir de la década del '70, con particular énfasis en el último período signado por los convenios urbanísticos.

\footnotetext{
${ }^{1}$ Recientemente, se han producido avances en algunas provincias del país en cuanto al alcance de los marcos normativos y las propuestas de planificación territorial, teniendo como ejemplo las recientes promulgaciones de la Ley de acceso justo al hábitat N¹4.449 en la provincia de Buenos Aires (Provincia de Buenos Aires, 2012) y la Ley de ordenamiento territorial Nº 8051 en Mendoza (Provincia de Mendoza, 2009); aunque ambas se encuentran en una etapa de incipiente implementación.
} 


\section{MARCO GENERAL DE LA INVESTIGACIÓN}

\subsection{Marco conceptual}

Este trabajo tomará como marco general los conceptos desarrollados alrededor de la noción de producción del espacio (Lefebvre, 2013 \{1974\}) en el contexto del urbanismo neoliberal (Peck, Theodore, \& Brenner, 2013; Theodore, Peck, \& Brenner, 2009) en diálogo con los autores que han conceptualizado las estrategias de reestructuración espacial (Harvey, 1989, 2007a) y los procesos de urbanización (Indovina, 2007; Janoschka, 2002).

Hacia principios de la década del ' 70 , Lefebvre introducía un cambio de paradigma a la hora de pensar el espacio urbano, afirmando que era inescindible de cualquier análisis de la ciudad el reconocimiento y comprensión del proceso de urbanización y configuración territorial, no ya como un elemento aislado y bajo un análisis descriptivo-morfológico sino bajo un análisis crítico de los procesos, para poder comprender lo que se produce, cómo y quiénes lo producen. Esta concepción motivó a entender al espacio urbano como resultado de las prácticas, relaciones e intereses de los grupos sociales, siendo a su vez producto que se comercializa, se utiliza y se consume; idea que se extendió a numerosas investigaciones alrededor del rol de la ciudad en el sistema capitalista. A modo de síntesis, desde la sociología Topalov desarrolló los conceptos en relación a la urbanización capitalista (1978); Horacio Capel (1974) desde la geografía, sostenía la tesis en torno a la producción del espacio español dentro del sistema capitalista. Los autores coincidían en que la ciudad y el espacio eran modelados en función de los intereses de ciertos agentes: los promotores inmobiliarios y empresas constructoras, generando nuevos patrones a través de sus operaciones en el espacio urbano y; los organismos públicos en su doble función de árbitros y agentes en el proceso de urbanización.

Paralelamente el neoliberalismo, se iniciaba como una respuesta estratégica y creativa a la crisis desatada por las políticas de Estado de bienestar y la disminución de la rentabilidad industrial sufrida por los países dominantes de Europa. Esta reconversión se basó en el desmantelamiento de los aparatos institucionales que habían mantenido las políticas económicas de base keynesiana para dar paso a políticas de gobierno que fortalecieran al mercado y la mercantilización de todos los ámbitos de la sociedad (Brenner \& Theodore, 2002). Para la década del ' 80 y '90 el modelo neoliberal se convirtió en la fuerza hegemónica tanto económica, como política e ideológicamente, que alcanzó y se afirmó en los países "periféricos" a través de programas de financiamiento o tratados comerciales con organizaciones internacionales como la OMC, el FMI o el Banco Mundial. Para los países latinoamericanos, los organismos de crédito internacional se convirtieron en agentes que institucionalizaron las "fuerzas del mercado" en la estructura de gobierno, intensificando las formas de intervención estatal a favor de imponer las reglas del mercado.

Los modelos de desarrollo propuestos bajo el paradigma neoliberal contienen al espacio urbano como pieza estratégica en su lógica de organización socioeconómica, entre los rasgos más importantes se destaca la incidencia de los procesos económicos sobre los patrones de urbanización, consolidado a través de las políticas urbanas, su marco regulatorio y el proceso de mercantilización de la ciudad. Estas nociones refuerzan el concepto desarrollado por Harvey (2007b) cuando expresa el carácter "creativo" de los cambios institucionalesespaciales, la destrucción-producción del espacio económico-político a través del proceso de neoliberalización y la noción de "arreglo espacial" para referirse a la resolución temporal de las crisis y contradicciones capitalistas mediante el desplazamiento a través del espacio. En este punto es fundamental reconocer su papel en el proceso de restructuración urbana que tiene sus matices de acuerdo al contexto de aplicación, determinados por sus marcos institucionales, prácticas regulatorias y luchas políticas. La distinción del carácter geo-político del neoliberalismo, nos permite acercarnos al concepto de "neoliberalismo realmente existente" (Brenner \& Theodore, 2002), que realza las interacciones existentes y contextualmente específicas entre los marcos regulatorios "autóctonos" y los proyectos de reestructuración neoliberal que se quieren aplicar.

En Latinoamérica, la acumulación y concentración del capital ha sido estructurante para las transformaciones urbanas como bien apuntan Jaramillo (2009) y De Mattos (2007, 2016); para ello se han provocado fenómenos simultáneos y convergentes que han reconfigurado las estructuras espaciales. Siguiendo nuestro marco conceptual, nos centraremos aquí en dos de ellos: un modelo de urbanización sostenido por políticas económicas neoliberales y la asociación de los gobiernos locales con el capital privado. Estos fenómenos han logrado instalar un orden urbano "empresarial", o como denomina Harvey (2007a) un "empresarialismo urbano" para referirse al crecimiento de las ciudades tanto en términos cualitativos como cuantitativos, dependiendo de las condiciones que ofrezcan las administraciones locales para la revalorización y circulación del capital, en el 
que se interpreta a la ciudad como bien de consumo y asumiendo el Estado un rol promotor inmerso en una lógica empresarial que ha reorientado las políticas públicas urbanas, las dinámicas económicas y la financiarización del capital. Claro está que para que estos fenómenos puedan sucederse, es necesario el acompañamiento del Estado a través de la liberalización, desregulación y la facilitación de operaciones que efectiviza la aplicación de la agenda neoliberal; no prescindiendo de él, sino limitando su accionar al uso de la fuerza, el control de las masas y la regulación de las prácticas para garantizar el "libre mercado". Esta asociación, es señalada por Harvey como "gobernanza urbana", conceptualizando la manera en que el poder político se vincula con los grupos empresarios en la administración gubernamental, modificando sus políticas a los fines de beneficiar la reproducción del capital.

En simultáneo, se ha generado extensa literatura académica (Lincoln Institute) e incluso oferta educativa desde agencias de financiamiento internacional (BID, BM, entre otros) como estrategia de promoción para alentar a los cuadros técnicos de los gobiernos locales a incorporar nuevos instrumentos de gestión fomentando un "modelo de gobernanza del suelo urbano".

Los instrumentos de gestión urbana si bien no son de aparición reciente, en las últimas dos décadas han tenido un fuerte auge en las administraciones locales latinoamericanas gracias a su configuración y nomenclatura específica en la normativa urbana, ajustada a los marcos jurídicos de cada contexto particular, como resultado de la lucha de intereses entre los agentes que producen el espacio urbano. Esta particularidad, unido al hecho de que han sido implementados y defendidos por diversas administraciones con heterogéneas orientaciones ideológicas, demuestra la maleabilidad de los mismos para responder a intereses muy diversos (Maricato \& Ferreira, 2002). Los aportes conceptuales en torno a los instrumentos destacan su neutralidad técnica dada por su capacidad de adaptación a diferentes condiciones de aplicación, aunque polarizando las posiciones entre aquellos que defienden los beneficios de su uso más allá de las condiciones macro económicas y políticas, y otros que tienen sus recaudos respecto del impacto nocivo de su empleo bajo condiciones poco claras, destacando su dependencia a exigencias políticas más que técnicas.

\subsection{Marco de antecedentes}

En este trabajo intentaremos realizar una lectura actualizada con los aportes conceptuales mencionados anteriormente. El boom de las interpretaciones marxistas-lefebrianas acerca de la ciudad y su rol en el sistema capitalista decayeron durante las décadas ' 80 y '90, los contextos de producción de las ideas y las prácticas estaban bajo nuevos paradigmas relacionados a la planificación estratégica y los efectos de la globalización en las áreas urbanas. Sin embargo, a mediados del 2000 resurgió una oleada de trabajos que retomaban las definiciones acerca de la influencia de los procesos económicos-financieros en la reestructuración espacial y los procesos urbanos, identificando el rol clave de la urbanización para el desarrollo del sistema neoliberal. Las investigaciones en relación a esto no han dejado de crecer desde entonces, diversificándose según las perspectivas de lectura. Enfocándonos en la literatura latinoamericana, dado el contexto específico del proceso de urbanización y las características de su producción, algunos se concentraron en la mercantilización de la ciudad (Abramo, 2012; De Mattos, 2016; Pírez, 2005, 2016); la relación entre ciudad, mercado y empresas (Baer, 2008; Baer \& Kauw, 2016; Capdevielle, 2014; Socoloff, 2013) o en el rol del Estado y las políticas públicas (Barenboim, 2012; Guevara, 2015).

La pregunta por la asociación de los gobiernos locales y agentes privados nos llevó a acerarnos a distintas formulaciones y aproximaciones respecto a su relación con los procesos de urbanización. Los APP pasaron a ser conocidas en el mundo por estar asociadas a novedosas formulaciones de políticas públicas, vinculadas a nuevos instrumentos de planeamiento y gestión urbana; las primeras experiencias se registraron por los ' 90 en Inglaterra y luego fueron replicadas por Europa y EE.UU. bajo la denominación de Public-Private Partnership, cuyo primer objetivo se basaba en la expansión de las inversiones estatales en obra pública sin aumentar la brecha de deuda, aumentando sus posibilidades de financiamiento a través de los agentes privados (Cota, 2010). Desde las ciencias sociales encontramos investigaciones locales en torno a las experiencias de APP en la CABA (Goicochea, 2014); en Brasil se produjeron investigaciones en torno al impacto de las operaciones urbanas consorciadas dispuestas en el Estatuto da Cidade (Holanda, Rosa, \& Pequeño, 2017; Maricato, 2010; Maricato \& Ferreira, 2002). Encontramos también estudios locales contemporáneos que profundizan en el conocimiento y conceptualización de los grandes proyectos urbanos (GPU) utilizando los APP como financiamiento de operaciones puntuales de "desarrollo urbano"; tales son los casos de Puerto Norte en Rosario y Puerto Madero en Buenos Aires (Barenboim, 2015; Cuenya \& Corral, 2011; Cuenya \& González, 2017). 
Por otro lado, se destaca el estudio de los instrumentos de gestión aplicados o la existencia de instrumentos específicos para la captación de plusvalía por parte del Estado (Amborski \& Smolka, 2003; Duarte \& Baer, 2013; Reese, 2003; Smolka, 2012).

Las exploraciones conceptuales y empíricas alrededor de la relación de los marcos regulatorios con las dinámicas empresariales y patrones de desarrollo territorial son escasas; referentes próximos son los trabajos de Vicuña del Río (2013) y Díaz Orueta (2013). Si nos enfocamos en el caso de estudio, los antecedentes respecto al análisis de los marcos regulatorios son realizados desde investigaciones con enfoques cercanos al rol del Estado, la planificación urbana y las políticas públicas (Caporossi, 2006; Colautti, 2013; Lemma, 2017; Marengo et al., 2006); sin embargo las vinculaciones público-privadas aplicadas en la gestión urbana, su influencia en el proceso de urbanización y en la dinámica de producción del espacio no han sido profundizadas en nuestro ámbito.

Las experiencias de APP en relación a la creación de suelo urbano en Argentina son recientes, se encuentran documentados tres casos de aplicación: Córdoba (ord. 12.077/12), Rosario (ord. 8973/12) y Tandil (ord. 12679/11) y una tendencia a reproducir este mecanismo por otros municipios (como Funes y Rafaela en Santa $\mathrm{Fe}$ ). La particularidad de estos casos reside en la institucionalización en el marco normativo local de los APP para la producción de espacio urbano combinado con condiciones similares a los instrumentos de recuperación de plusvalía operados en otras experiencias.

\section{METODOLOGÍA}

Se resolvió adoptar como metodología para esta ponencia la revisión de la bibliográfica existente, de la legislación urbanística específica y de los APP aprobados, realizando una descripción y un análisis comparativo (no exhaustivo) de las asociaciones horizontales desde la década del 70, sin embargo, se concentrará mayor información y profundidad en las vinculaciones relacionadas a la última generación de APP. Para ello se procurará el análisis de contenido de información estadística, documentos oficiales (normativas, planes) y no oficiales (artículos periodísticos, publicitarios) relacionados con la temática. Se pretende exponer la influencia de las vinculaciones público-privadas en los procesos de producción del espacio mediante la comparación del vínculo entre a) las políticas públicas a través de los marcos normativos e institucionales y las estrategias regulatorias; b) los modelos de desarrollos propuestos, c) los actores que promueven los proyectos hegemónicos y d) las estrategias desplegadas por los desarrollistas inmobiliarios a escala local.

\section{CASO DE ESTUDIO}

Si bien los autores que dan sustento teórico a esta investigación, plantean que el neoliberalismo y el modelo empresarialista son procesos multi escalares y multi direccionales, el alcance de este trabajo se concentrará en la escala local, dada la particularidad en el contexto de aplicación: la autonomía municipal de los gobiernos locales para la legislación de las normas que regulan los procesos de creación y uso del suelo urbano, y en particular a la ciudad de Córdoba como caso de estudio. Esta decisión viene acompañada también por la comprensión de que, dada la condición de autonomía, las vinculaciones horizontales y su influencia tanto en el proceso de urbanización como en las condiciones de producción del espacio serán específicas a las condiciones contextuales generadas por cada gobierno local. Lo singular de este proceso intentará servir a aquellos otros similares a los que pueda ser útil su caracterización y conceptualización.

\subsection{Las condiciones locales}

La ciudad de Córdoba es la segunda urbe mayor poblada de la Argentina con una población de 1.329.604 habitantes, según el último censo oficial (INDEC, 2010) y conforme datos oficiales, cuenta con un ejido de 576 km2 -57.600 ha- coincidente con los límites políticos del departamento Capital, lo que determina una densidad de $2.308 \mathrm{hab} / \mathrm{km}^{2}$. A su vez, es la cabecera del aglomerado Gran Córdoba que representa al área metropolitana generada por la conurbación de ciudades aledañas más pequeñas. 


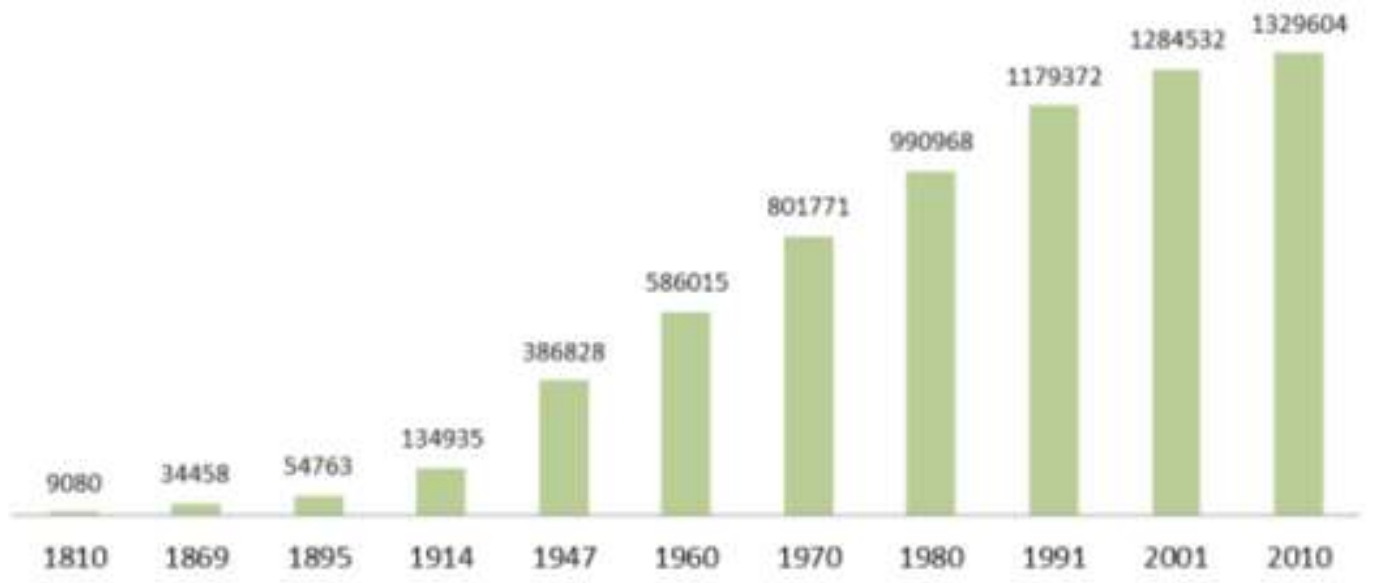

Gráfico 1: Evolución del crecimiento de la población de la ciudad de Córdoba. 1810- 2010. Absolutos. Fuente: elaboración propia en base a datos del INDEC. Censos nacionales de población, hogares y viviendas.

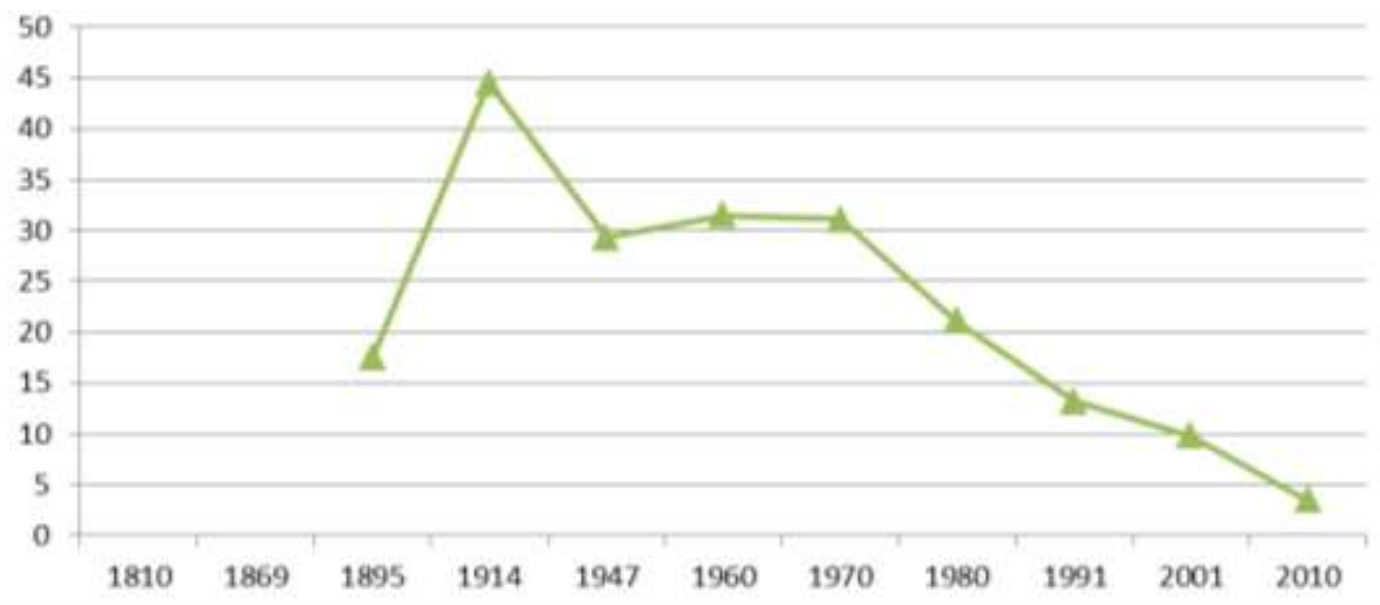

Gráfico 2: Evolución de la tasa media de crecimiento inter censal. Ciudad de Córdoba. 1895-2010.

Fuente: elaboración propia en base a datos del INDEC. Censos nacionales de población, hogares y viviendas.

\begin{tabular}{|l|c|c|c|c|c|}
\hline Localidades & $\mathbf{1 9 9 1}$ & $\mathbf{2 0 0 1}$ & $\mathbf{2 0 1 0}$ & Crecimiento 2001-2010 (en \%) & Distancia \\
\hline Córdoba & 1.179 .372 & 1.284 .532 & 1.329 .604 & $3,5 \%$ & - \\
\hline Villa Allende & 16.025 & 21.683 & 28.374 & $30,9 \%$ & $16 \mathrm{~km}$ \\
\hline Malagueño & 5.866 & 6.404 & 13.102 & $104,6 \%$ & $20 \mathrm{~km}$ \\
\hline Mendiolaza & 1.536 & 4.204 & 10.317 & $145,4 \%$ & $20 \mathrm{~km}$ \\
\hline Rio Ceballos & 12.802 & 16.632 & 20.242 & $21,7 \%$ & $32 \mathrm{~km}$ \\
\hline Saldan & 1.868 & 2.099 & 10.606 & $405,3 \%$ & $34 \mathrm{~km}$ \\
\hline
\end{tabular}

Tabla 1: Evolución de la cantidad de población por localidades seleccionadas de la región metropolitana de Córdoba. 1991-2010. Absoluto y relativo.

Fuente: elaboración propia en base a datos del INDEC. Censos nacionales de población, hogares y viviendas.

La ciudad ha tenido un constante crecimiento de población desde su fundación, aunque puede notarse un ritmo disímil de crecimiento en las tasas de evolución inter censal (Gráfico 2) que en la actualidad vienen marcando una tendencia decreciente, afectada principalmente por la movilización de la población hacia ciudades aledañas que integran su región metropolitana (Tabla 1).

Según un estudio del Atlas de Crecimiento Urbano de la Universidad Torcuato di Tella (Centro de Investigación de Política Urbana y Vivienda -CIPUV-, 2015), la ciudad incrementó en 7258 ha su territorio urbanizado en los últimos 20 años (1990-2010), lo que representa un 61\% si tomamos como base los registros de 1990. 


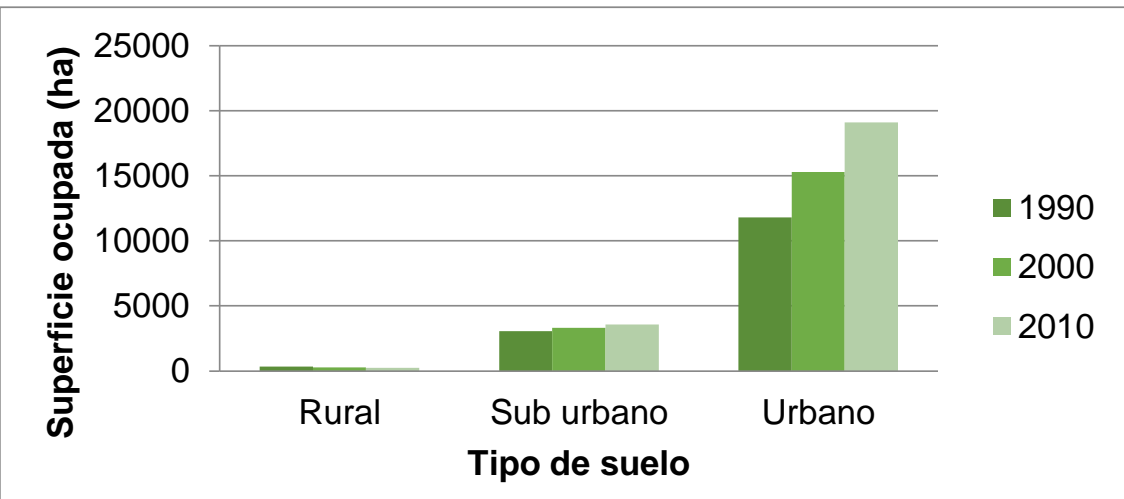

Gráfico 3: Evolución del tipo de ocupación del ejido municipal en los últimos 20 años. Ciudad de Córdoba, 2010. Absolutos. Fuente: Elaboración propia a través de datos del Atlas CIPUV de Crecimiento Urbano

\section{Indicadores}

Total de población (absolutos)

Estructura de la población por grupos etarios (en \%)

$0-14$

$15-64$

65 y más

Índice de masculinidad

Condición de actividad (en \%)

Ocupado

Desocupado

Inactivo

Viviendas (absolutos)

Localización (en \%)

Urbana

Rural agrupada

Tipo de vivienda (en \%)

Casa

Departamento

Rancho

Casilla

Calidad de la vivienda (en \%)

Calmat1

Calmat2

Calmat 3

Calmat 4

Hogares (absolutos)

Hogares con NBI (en \%)

Tabla 1: Indicadores socio demográficos seleccionados. Ciudad de Córdoba, 2010.

Fuente: elaboración propia en base a datos del INDEC. Censo nacional de población, hogares y viviendas, 2010.
Total de la ciudad

1.329.604

$23.55 \%$

$66.20 \%$

$10,25 \%$

91,23

$63,20 \%$

$4,87 \%$

$31,93 \%$

472.571

$99,24 \%$

$0,76 \%$

$70,4 \%$

$28,39 \%$

$0,4 \%$

$0,30 \%$

$54,68 \%$

$41,34 \%$

$3,15 \%$

$0,83 \%$

\subsection{7}

$5,82 \%$ 


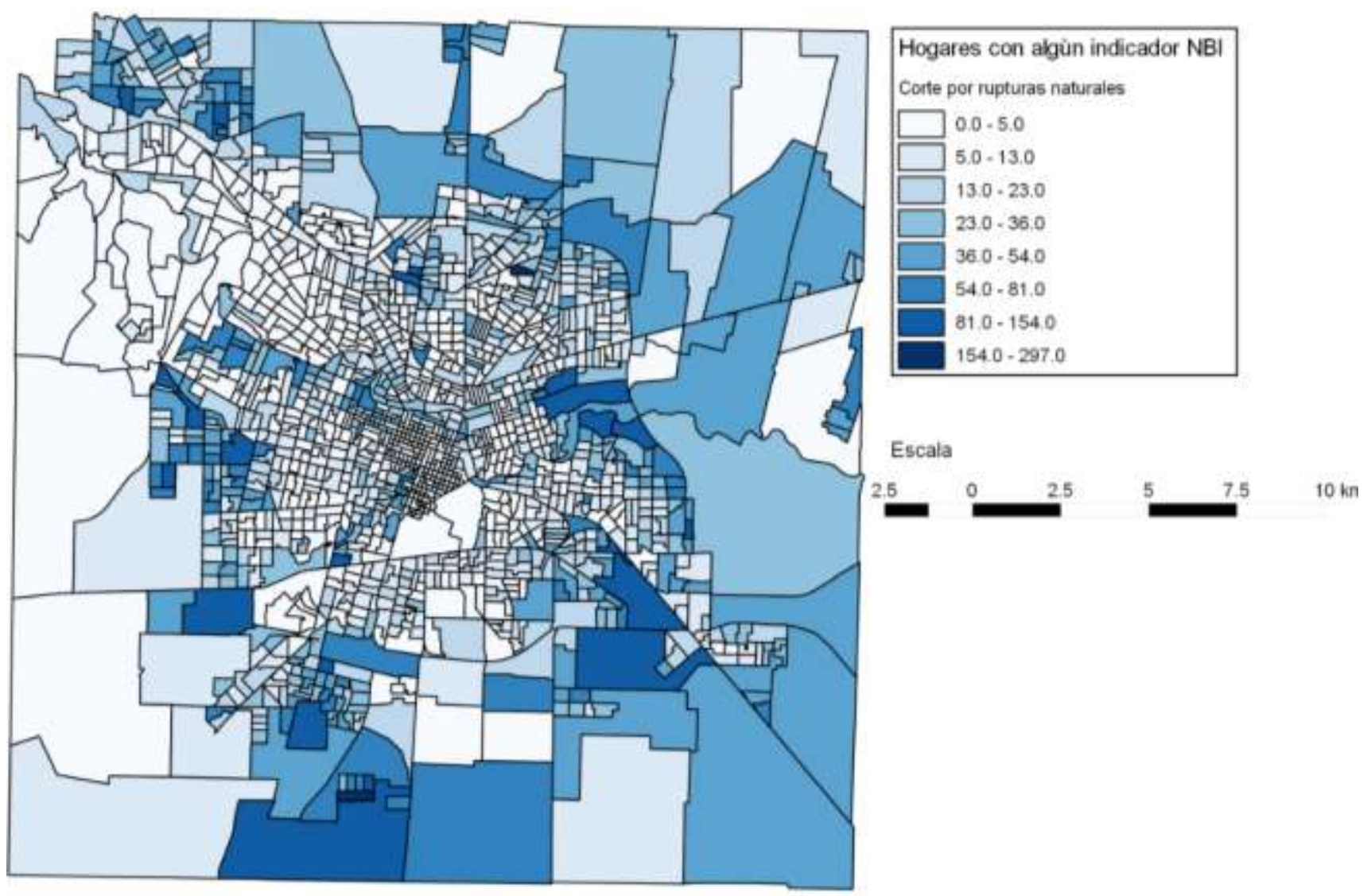

Gráfico 4: Geo referenciación, cantidad de hogares con algún indicador NBI según radios censales. Ciudad de Córdoba, 2010. Fuente: elaboración propia en base a datos del INDEC. Censo nacional de población, hogares y viviendas, 2010. Datos procesados con Redatam+SP. Base geográfica disponible en http://www.indec.gob.ar/codgeo.asp

\subsection{Las tendencias en la gestión urbana local}

La conversión del Estado de bienestar por el modelo de acumulación capitalista adoptó bases más flexibles tanto para la normativa urbana como para los modos de vinculación entre el poder público y el sector privado. Esta condición global, no despoja a las APP de las limitaciones y especificidades propias de las condiciones locales como bien hemos resaltado anteriormente, confirmando aquella idea de que el espacio urbano, como espacio producido socialmente, es resultado de los conflictos e intereses entre los agentes que lo producen configurando una lógica propia; un proceso urbano específico para cada contexto geográfico e histórico que incluye la formulación y adopción particular de las políticas urbanas y con ello los APP. Nos enfocaremos en este estudio en las variaciones que se han ido admitiendo en la actuación del gobierno local y sus asociaciones, proceso que ha ido constituyendo hasta la actualidad la institucionalización de los APP en el marco normativo, consolidado como instrumento de gestión urbana bajo la denominación de "convenios urbanísticos" para distinguir patrones de aplicación y su vinculación con el proceso de producción del espacio.

La década del '60 en nuestro país, transcurrió entre golpes de Estado e intentos de recuperación de la democracia signada por el contexto internacional de inestabilidad política-económica en los países centrales y de guerra fría que veía en Latinoamérica un territorio disputado simbólica y económicamente.

Para los '70, en el campo del urbanismo latinoamericano lo que se traspuso se condensaba en tres tendencias: la urbanización dependiente, la visión estructuralista marxista y los funcionalistas (apoyada en una red de instituciones como la Unesco, Cepal, Siap y fundaciones como Rockefeller y Ford con financiamiento norteamericano interesadas en el control social). Claro está, dado el contexto nacional, la postura adoptada fue esta última, dejando como herencia un dogma de "liberalización" de la administración pública acorde a estos postulados que tuvo su correlato en la planificación urbana. En la ciudad, se construye el documento "Diagnóstico tentativo y alternativas de desarrollo físico para la ciudad de Córdoba" desde la asesoría de planeamiento urbano de la Municipalidad, en el que se expresa preocupación por la extensión de la mancha urbana aunque se asume el crecimiento como resultado del "libre juego de las fuerzas económicas y sociales" 
con escasa participación del Estado (Caporossi, 2006). A partir de este documento, podemos decir que se genera un modelo de crecimiento de la ciudad, enfocado en el sistema físico y vial de la estructura urbana que se desarrolló a partir de "ejes preferenciales de desarrollo" tal como se indicaba en el modelo teórico, aprobado mediante ordenanza municipal en 1978 como "Esquema de ordenamiento urbano para la ciudad de Córdoba: año 2000" (EDOU) y luego aparecerá reafirmado en el "Esquema Director de Ordenamiento Metropolitano (EDOM)“ en 1979 (Lemma, 2017). Como expresión de estas decisiones, intensificadas por la insuficiencia de servicios e infraestructura en la creciente ocupación periférica y dadas las dificultades con que se enfrentaban los gobiernos ante la crisis económica internacional, primaron los acuerdos para la inversión pública destinadas a la construcción de infraestructura vial y la red de servicios urbanos, mediante la modalidad de licitación: obra pública financiada por recaudación fiscal específica combinada con toma de financiamiento externo y ejecución de empresas privadas.

En la década del ' 80 , se produce una fuerte desaceleración en la ejecución de obra pública en general, sumado a esto la producción del espacio urbano fomentada por las "fuerzas económicas" evidenciaba su autonomía de la planificación urbana. Por ello, se intentó regular el accionar privado a través de un conjunto de normas que imponían limitaciones y determinaban condiciones a los mecanismos de creación de suelo urbano, evidenciando tensiones en el control del privado por parte del poder público; elaborando el cuerpo normativo que reemplaza al EDOU y regulará el uso y fraccionamiento del suelo y una detallada normativa particular para el área central que, con modificatorias, se encuentra vigente actualmente. El modelo físico que se adopta (Ordenanzas 8057/85; 8060/85; 8056/86) definirá tres áreas urbanas a modo de anillos concéntricos: área central -núcleo central histórico-; intermedio-barrio tradicional- y periféricos, correspondiendo al modelo de "zoning" y respondiendo fielmente al modelo de renta (Caporossi, 2006), a partir de áreas urbanizables, usos y densidades habilitadas para el aprovechamiento del máximo potencial de la infraestructura instalada y limitación al avance de suelo urbano sobre áreas rurales. Sin embargo, los mecanismos para la reproducción de capital son ingeniosos y los resultados de la restricción normativa fueron nuevas estrategias de los agentes privados para la producción de suelo urbano, usando "creativamente" el marco regulatorio y consolidando las tendencias de centralización de servicios y densidad, corredores viales de usos mixtos y periferia extendida de baja ocupación.

En los '90, se propusieron documentos que no llegaron a implementarse en su totalidad, pero que generaron las líneas de desarrollo posteriores. Para 1996, se elabora el Plan estratégico para la ciudad (PEC) configurando el proceso de descentralización administrativa, considerando fuertemente los aportes de la planificación estratégica. Acompañando este proceso de generación de "nuevas centralidades-CPC-", se produjo una fuerte liberalización del suelo, desviando la atención desde los aspectos morfológicos hacia concepciones flexibles de la producción del espacio. Las asociaciones se direccionaron a la flexibilización normativa y se materializaron en la ciudad a través de la extensión de la urbanización sobre suelo rural a baja densidad y el crecimiento periférico por la localización selectiva de nuevas tipologías privadas (ORD. 8606/91 para urbanizaciones cerradas de elite incorporando las urbanizaciones residenciales especiales - URE).

Ya para la década del 2000, el gobierno local retoma la intención de generar un plan urbano, representado por varios documentos (Diagnóstico y líneas estratégicas para el Plan Urbano Ambiental-2001; Formulación de lineamientos alternativos para el plan urbano ambiental-2003; Plan Estratégico para la ciudad de Córdoba. PECba-2005; Bases para el Plan Director de la Ciudad de Córdoba 2020-2008), que otra vez no lograron implementarse. En referencia al proceso de urbanización y las modalidades de producción se produjeron una serie de transformaciones del marco regulatorio, cristalizando el formato de gobierno asociado a la economía privada que se traslucieron en una generación de propuestas centradas en zonas específicas de la ciudad, en las que se aceptó la participación de inversionistas privados con proyectos puntuales y con objetivos a corto plazo (ORD.10988/05-Portal del Abasto). Paralelamente, bajo esta coyuntura privatista, se conformaron las corporaciones inmobiliarias que dieron lugar a los grandes proyectos urbanos, el agrupamiento de los desarrollistas enmarcados como gremio a través de la C.E.D.U.C. ${ }^{2}$ desde 2003 dio lugar a la penúltima generación de emprendimientos inmobiliarios. Simultáneamente, la participación de los gobiernos locales en la imposición de la lógica del capital privado sobre los procesos urbanos se expresaba a través de las

\footnotetext{
2 "Cámara Empresarial de Desarrollistas Urbanos Córdoba" -CEDUC-cuya misión institucional es "la promoción, progreso y protección de la actividad desarrolladora urbana privada, en la ciudad y la provincia" tal lo expresa su página web (http://www.ceduccba.com.ar/ceduc/. Fecha de consulta 20/02/2016.
} 
modificatorias ex post facto al marco normativo y en acciones ejecutadas por políticas públicas. ${ }^{3}{ }^{4}$ Esta producción se caracterizó por un lado, por la consolidación de un nuevo estilo de urbanización, los barrios cerrados periféricos, a través de la reproducción del estatus social tanto de clase alta como de clase baja, diferenciándose del resto de la ciudad aludiendo cuestiones de seguridad y calidad ambiental; y por otro lado, la reducción discrecional de exigencias normativas para la ejecución de tipologías comerciales o residenciales derivadas del modelo privatista.

Para la década actual, en 2015 aparece la ordenanza denominada "Modelo de producción de ciudad- Áreas de promoción urbana" (Ord. 12483/15) que modifica numerosos artículos de la vieja norma de ocupación y usos del suelo del ' 86 con el objetivo institucional de "definir políticas que promuevan acciones de transformación urbana y adecuar la regulación del desarrollo urbano a la realidad socio económica y política actual”; aunque conviviendo en parte con aquella. Paradójicamente, el modelo no incluye las modificaciones producidas en la ocupación del suelo por la utilización de la ordenanza № 12.077 de Convenios Urbanísticos (2012).

1810

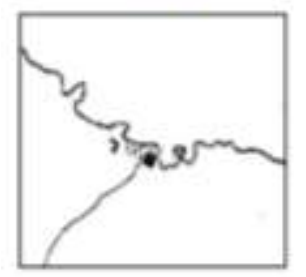

Poblacioben 9.000

1991

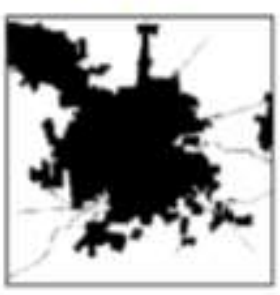

Potiacion 1.170.372
1870

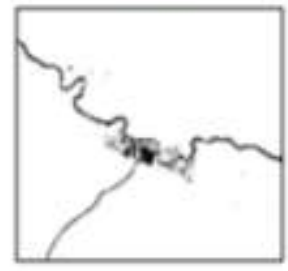

Pobtacion 36.223

2001

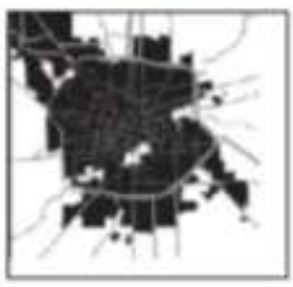

Poblaciben $1.284 .5 e 2$
1900

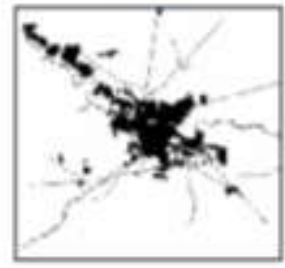

Poblaciôn 72500

2010

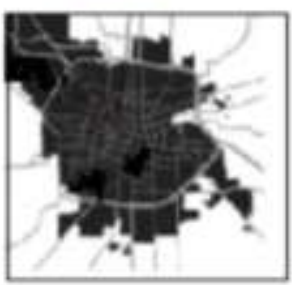

Poblacion 1.329 .604
1960

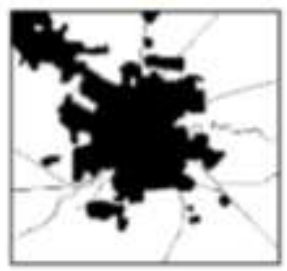

Potalación 509.163
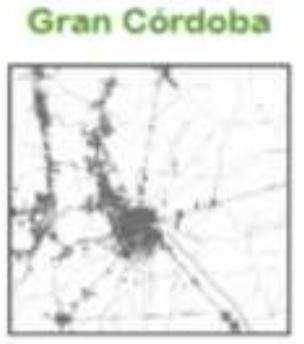

Población 1.9e1.737

Gráfico 5: Evolución de la población y crecimiento de la mancha urbana. Ciudad de Córdoba, 1810-2010.

Fuente: Elaboración propia según Municipalidad de Córdoba. Secretaría de planeamiento e infraestructuras. Subsecretaría de planeamiento.

\subsection{Los APP y las últimas generaciones de emprendimientos inmobiliarios: los convenios urbanísticos en Córdoba}

El gobierno municipal ha invertido cada vez más esfuerzos en asociaciones con el sector privado, apoyándose en un discurso de que los fondos públicos no son suficientes para las obras necesarias, y que es imprescindible el aporte de los inversionistas privados; quienes a su vez afirman abiertamente su capacidad de "hacer ciudad", influyendo en las decisiones públicas por su interés e idoneidad en la producción del espacio urbano (Gargantini \& Peresini, 2017). La última generación de emprendimientos inmobiliarios se vincula a la aplicación de los APP bajo el formato de "convenios urbanísticos", un instrumento de reglamentación de acuerdos que posibilitan la excepción normativa en lo relacionado con el fraccionamiento, uso y ocupación del suelo de predios ubicados en el ejido municipal como estrategia para la creación de suelo urbano, otorgando el rol

\footnotetext{
${ }^{3}$ La municipalidad de Córdoba, sancionó modificaciones (Modif. № 10760/04) a la Ordenanza № 8606/91 de usos del suelo para incorporar a la Urbanización Residencial Especial (URE), los barrios cerrados o "countries" que ya se habían instalado en la ciudad por fuera de la norma.

${ }^{4}$ Plan provincial de vivienda para la erradicación de villas "Mi casa, mi vida".
} 
creador a agentes privados. En su objetivo aparecen algunos de los principios rectores de las prácticas de asociación horizontal, tales como posibilitar mecanismos jurídicos, administrativos y económicos-financieros para favorecer el desarrollo de la ciudad; promover la inversión privada y alcanzar transformaciones urbanísticas y estructurales con mayor rapidez de ejecución y menor afectación de recursos públicos. Queda omitido en la norma, establecer áreas estratégicas o prioritarias para la implementación de este instrumento, dejando a merced del mercado inmobiliario su localización; como tampoco se establece la necesidad de cercanía con áreas urbanizadas, a costa de suelo barato que pueda convertirse en urbano. La ordenanza cuenta con 31 artículos, de los cuales sólo dos se destinan a especificar la disposición del beneficio a favor del gobierno local por estas excepciones, estableciendo las formas de "integración de la participación municipal", negociadas para cada caso.

A partir de su implementación, la ordenanza tuvo un fuerte impacto en un periodo muy corto, de los 23 iniciadores privados se concretaron 22 acuerdos entre 2012 y 2015 (el único excluido tenía graves irregularidades con los títulos de los lotes) y a partir de 2016 no se registraron nuevos, sino adendas a los ya acordados con numerosas controversias y resistencia ciudadana. ${ }^{6}$ Los 10 primeros convenios fueron celebrados con grandes empresas desarrollistas de la ciudad, todas integrantes de la CEDUC, y algunas de ellas han logrado más de un acuerdo.

Según datos oficiales, se han incorporado al suelo urbano 1.730 hectáreas del ejido, antes destinada a uso rural o industrial en el que se ha producido un salto de renta notable gracias a las excepciones "ad hoc", sufriendo el mayor impacto geográfico la zona sur, albergando ahora 1.580 ha más de tierra urbanizable. La participación del municipio por estos beneficios se compone principalmente de obras viales e infraestructura tendientes a mejorar la accesibilidad de los mismos emprendimientos beneficiados por los acuerdos; ${ }^{7}$ sesión de fracciones de tierra, materiales y/o dinero para viviendas destinados al "Plan de Emergencia Habitacional" del cual se desconocen aplicaciones; al "fondo de reparación urbanística"; equipamientos sociales a ser determinadas por el Municipio y "donaciones" al dominio público municipal de polígonos de tierra destinados a espacios verdes y calles dentro de los mismos emprendimientos afectados por el convenio. Por otro lado, en la ordenanza se dispone un plazo no mayor a dos años para la iniciación de las obras acordadas, luego de ese tiempo, el municipio podrá resolver el acuerdo sin que esto genere reclamo alguno. A la fecha, existen al menos 4 convenios que sobrepasan los 4 años de antigüedad sin inicio de las obras establecidas en el acuerdo pero con avances respecto al desarrollo inmobiliario. ${ }^{8}$

El proyecto nace "inspirado" en otros instrumentos de recuperación de plusvalía como argumento válido para los cambios normativos y el desarrollo urbano, destacando que todo el territorio del ejido es factible de "desarrollar" con un proyecto inmobiliario de cualquier envergadura, con el fin de incrementar la oferta de suelo para evitar la sangría de población hacia las ciudades conurbadas. Las estrategias de los desarrollos con mayor superficie e impacto ha sido la de fraccionar la oferta en "urbanizaciones" diferenciadas con nombres propios, siguiendo los distintos segmentos de poder adquisitivo, ofreciendo loteo uniformes que contienen terrenos desde $250 \mathrm{~m} 2$ a $1600 \mathrm{~m} 2$. En algunos casos se incluye también la oferta de viviendas, argumentando incluso en el mismo convenio, la conveniencia de aumentar la densidad poblacional prevista y desarrollar una "urbanización integral" que incluya todos los servicios (educación, religión, seguridad, recreación y comercios) pensando y publicitando una "nueva ciudad basada en valores comunitarios", con nombre e identidad propia, despegándose de los males que aquejan a la "otra" ciudad. Siguiendo estos lineamientos, la "nueva oferta"

\footnotetext{
${ }^{5}$ En cualquier tipo de combinación de dinero en efectivo; transfiriendo al dominio municipal un porcentaje de la superficie neta urbanizada, de acuerdo a la Ordenanza № 11.988: Banco de inmuebles; mediante la ejecución de obras de infraestructura, equipamientos sociales, u otras. Cabe aclarar que la Ord 11988 fue derogada en 2016 y con ella caducaron los "beneficios para el municipio". http://publicadord7.diaadia.com.ar/cordoba/sin-consenso-para-el-banco-de-inmuebles

6 http://www.lavoz.com.ar/opinion/convienen-los-convenios-urbanisticos; http://Imdiario.com.ar/noticia/16464/gano-un-millonario-perdimos-los-cordobeses http://www.diarioalfil.com.ar/2013/11/14/urbanistas-municipales-argumentaron-contra-mega-country-que-impulsa-mestre/ 7 http://www.cba24n.com.ar/content/circunvalacion-\%C2\%BFintereses-privados-en-medio-de-la-discusion

http://www.lavoz.com.ar/ciudadanos/denuncian-que-en-vez-de-parque-habra-lagunas-de-retardo\#!/registro https://www.infonegocios.info/nota-principal/grupo-proaco-salta-a-las-grandes-ligas-lanza-docta-una-ciudad-de-200hectareas

${ }^{8}$ http://www.lavoz.com.ar/ciudadanos/mestre-termina-el-ano-con-28-de-las-promesas-cumplidas http://www.lavoz.com.ar/politica/por-convenios-autorizaron-urbanizar-1741-hectareas

http://diaadia.viapais.com.ar/cordoba/apuran-nueve-convenios-urbanisticos-para-financiar-obras http://www.lavoz.com.ar/ciudadanos/siguen-sin-arrancar-nueve-obras-importantes-de-la-concertacion http://www.lavoz.com.ar/ciudadanos/un-batallon-de-beneficios-que-tardan-en-llegar
} 
producida a través de estos APP se concentra en segmentos de la población con altos ingresos y capacidad de pago, que en vez de adquirir un lote en un barrio privado del Gran Córdoba puede hacerlo en la ciudad.

Los beneficios otorgados a los emprendimientos no se limitaron sólo a los cambios normativos sino que posteriormente, y frente a la coyuntura nacional de apertura del mercado de créditos hipotecarios, se realizaron excepciones a los requerimientos para la escrituración, facilitando y promoviendo el mercado inmobiliario de los emprendimientos. ${ }^{9}$

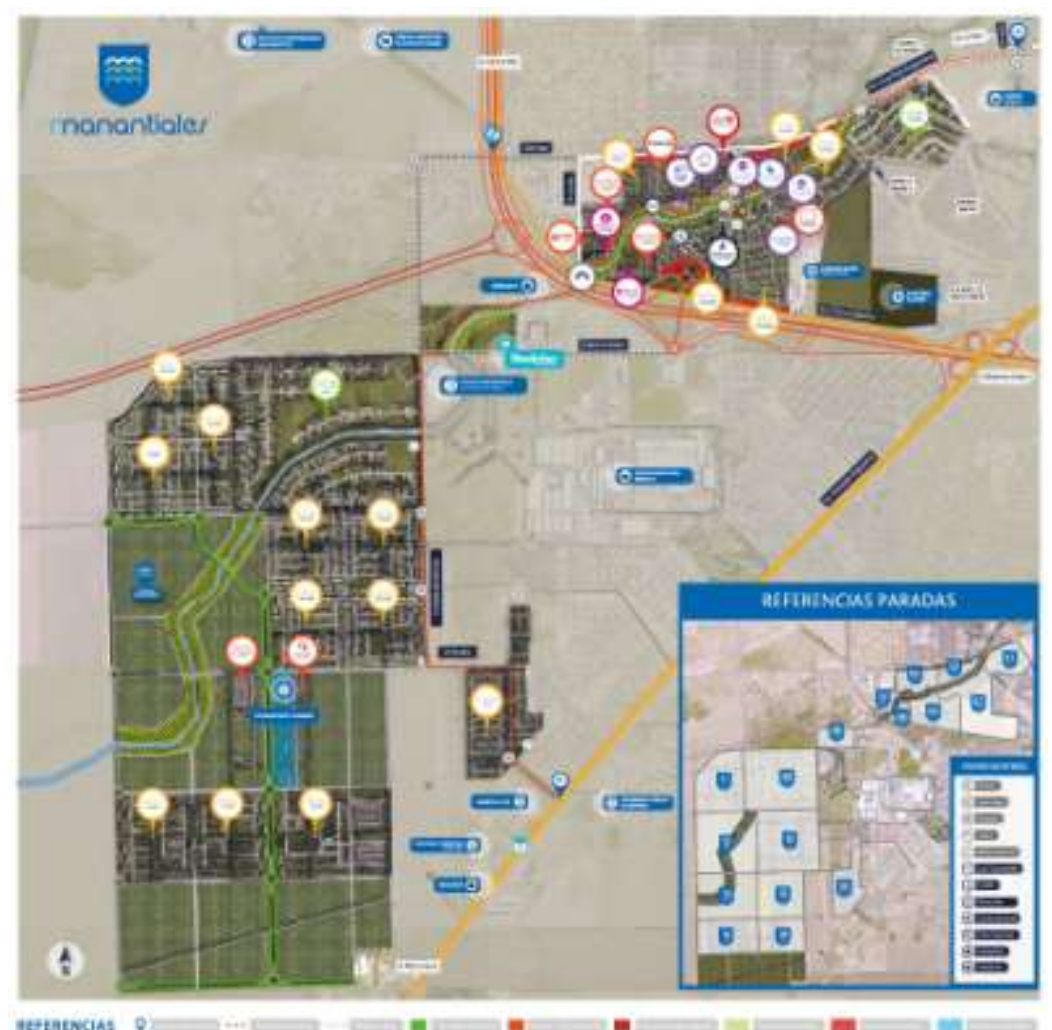

Gráfico 7: Mapa publicitario de desarrollo inmobiliario. "Ciudad Manantiales".

Fuente: Elaboración propia a partir de página web Grupo Edisur. http://www.grupoedisur.com.ar/web/es/manantiales-360/

\footnotetext{
${ }^{9}$ https://infonegocios.info/nota-principal/mestre-y-cossar-ultiman-una-ordenanza-para-liberar-30-000-lotes-para-escriturardesarrollistas-felices
} 


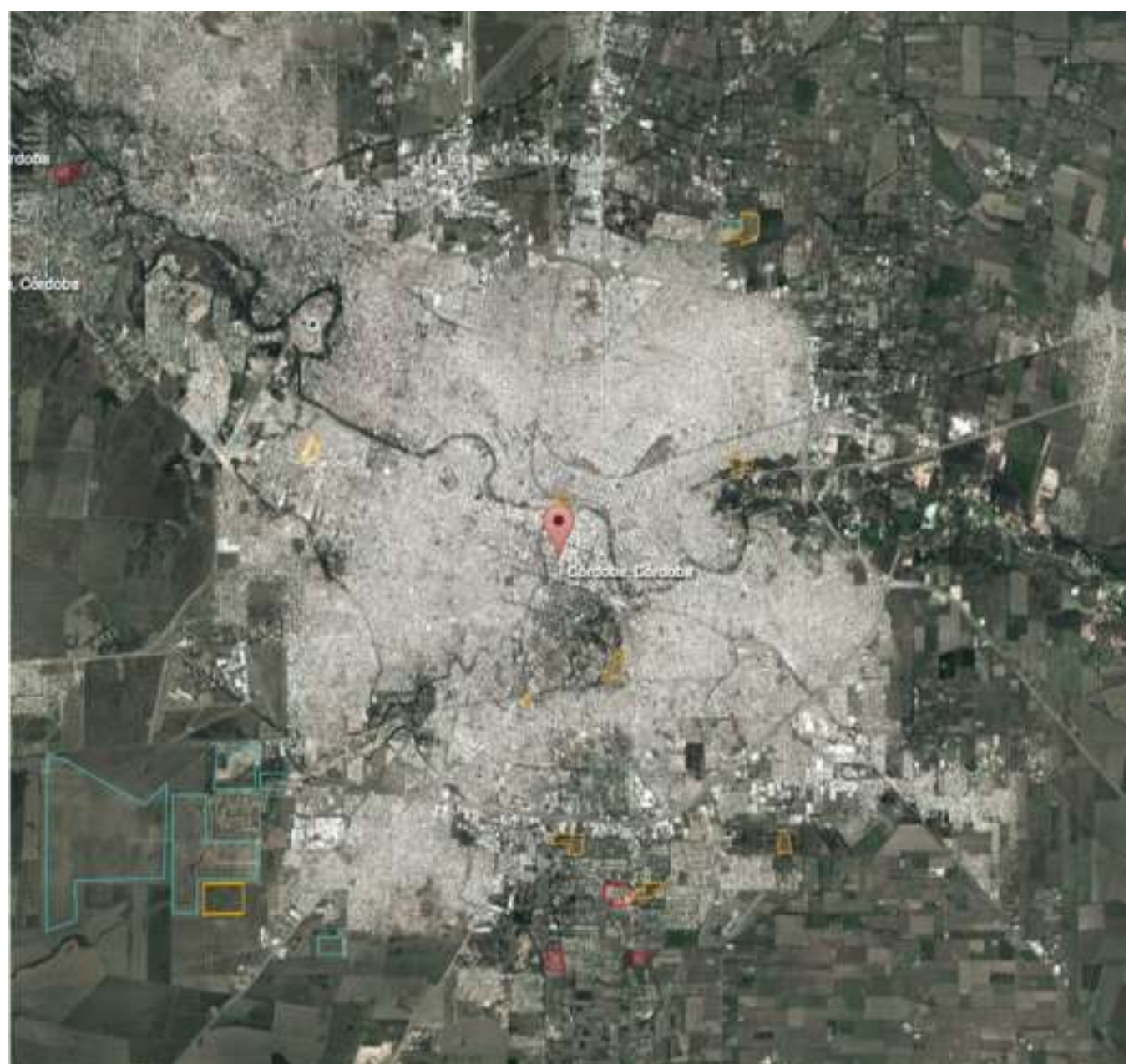

Gráfico 6: Geo localización de convenios urbanísticos aprobados en el período 2012-2016. Ciudad de Córdoba.

Referencias: En línea celeste: Convenios aprobados hasta 2014. En línea morada: Convenios aprobados hasta enero de 2015. En línea naranja: Convenios aprobados post enero de 2015.

Fuente: Elaboración propia en base a datos de la Municipalidad de Córdoba y digesto municipal.

\section{A MODO DE CIERRE}

A partir de este escueto recorrido normativo-institucional podemos determinar algunas cuestiones importantes. En primer lugar, es posible reconocer cambios institucionales en el proceso de urbanización y planificación de la ciudad que se relacionan con los procesos políticos y macroeconómicos, sustentando la relación entre las transformaciones urbanas y los sucesivos momentos de "destrucción creativa". Por otra parte, el proceso, y a pesar de sus contradicciones y superposiciones, nos demuestra la evolución de distintas modalidades de interacción entre el gobierno local y los actores privados en el ámbito de la gestión urbana a través de numerosos ajustes técnicos-administrativos-normativos, hasta llegar a la actualidad con la institucionalización clave de los APP en "convenios urbanísticos".

En segundo lugar, la explicitación del vínculo Estado-privado a partir de dicha institucionalización, que se presenta como un instrumento de gestión urbana novedoso y oportuno, puede ser leído también como un avance más hacia un modelo de relación horizontal estratégico entre el gobierno local y el sector privado en el marco de un Estado empresarialista que revaloriza el papel de la actividad inmobiliaria y considera a sus actores como agentes preponderantes, respecto al resto de los actores sociales involucrados, en la producción del espacio urbano.

Sobre el papel de los APP en la gestión urbana, en su contexto y aplicación en el caso de estudio, podemos decir que los "beneficios para el municipio" en estos acuerdos han sido calculados y establecidos en claro beneficio del agente privado: los cambios otorgados han provocado diferencias sustanciales en el rendimiento y rentabilidad del suelo y se han utilizado como garantía de rentabilidad para los activos invertidos por los 
agentes privados; no existe control alguno de las contraprestaciones acordadas, e incluso, se desconocieron los beneficios que se habían destinado al banco de inmuebles; las urbanizaciones generadas no responden a la demanda real de la población sin acceso a la tierra o a la vivienda; se desatienden e ignoran los reclamos ciudadanos en relación al impacto que producirían estos emprendimientos. En síntesis, la aplicación de estos APP ha sido ampliamente utilizada para la demanda específica de los desarrollistas inmobiliarios, asegurando su expansión territorial y de capital, contradiciendo las motivaciones discursivas en torno a la recuperación de plusvalía y la intervención del Estado en la renta del suelo urbano, redefiniendo los propósitos de este instrumento y de la gestión urbana con fines políticos antes que económicos. Ante estas contradicciones entre los discursos y las prácticas, podemos representar aquí una nueva fase de la "neoliberalización de la gestión urbana" que tiene a los APP como dispositivo de consolidación.

\section{BIBLIOGRAFÍA}

Abramo, P. (2009). La producción de las ciudades latinoamericanas: mercado inmobiliario. (F. Carrión, Ed.). Quito, Ecuador: Organización Latinoamericana y del Caribe de Centros Históricos (OLACCHI).

-- (2012). La ciudad com-fusa: mercado y producción de la estructura urbana en las grandes metrópolis latinoamericanas. EURE (Santiago), 38(114), 35-69.

AMBORSKI, D., \& SMOLKA, M. (2003). Recuperación de plusvalías para el desarrollo urbano: una comparación inter-americana. EURE-Revista Latinoamericana de Estudios Urbanos Regionales, XXIX, 55-77.

BAER, L. (2008). Crecimiento económico, mercado inmobiliario y ausencia de política de suelo. Un análisis de la expansión del espacio residencial de la Ciudad de Buenos Aires en los 2000. Revista Proyección, 2(5), 245.

BAER, L., \& KAUW, M. (2016). Mercado inmobiliario y acceso a la vivienda formal en la ciudad de Buenos Aires. Eure, 42(126), 5-25.

BARENBOIM, C. (2012). Politicas publicas urbanas e instrumentos de regulación. Revista lberoamericana de Urbanismo, 7, 31-41.

-- (2015). Análisis de los instrumentos de regulación urbana empleados en Puerto Norte y sus áreas de influencia, en Rosario, Argentina. Revista Ciudades, Estados Y Política, 2(1), 1-14.

Brenner, N., \& TheOdore, N. (2002). Cities and the Geographies of "Actually Existing Neoliberalism." Antipode, 34(3), 349-379.

CAPdeVielle, J. (2014a). Espacio urbano y desigualdades: las políticas públicas y privadas en la ciudad de Córdoba. Cuadernos Geográficos, 53(2), 135-158.

-- (2014b). Los grupos "desarrollistas" y su incidencia en el espacio urbano de la ciudad de Córdoba, Argentina (1990- 2013). Terra Nueva Etapa, XXX, 129-152.

CAPEL, H. (1974). Agentes y estrategias en la producción del espacio urbano español. Revista de geografia [en línia], Vol. 8, Núm. 1, p. 19-56.

CAPorossi, C. (2006). Planificación y crecimiento urbano. Ideas y reflexiones a partir del caso de la ciudad de Córdoba., En La periferia de Córdoba. Cuestiones sobre el hábitat. (pp. 22-40). Córdoba: Departamento de publicaciones FAUD - UNC.

ColaUtTI, V. (2013). Procesos de transformación urbana en la ciudad de Córdoba. V Seminario Internacional de Investigación en Urbanismo, Barcelona-Buenos Aires, junio 2013 (pp. 480-495). Barcelona: DUOT.

COTA, D. A. (2010). A parceria público-privada na política urbana brasileira recente: reflexões a partir da análise das operações urbanas em Belo Horizonte [Tese. Doutorado. Geografia] (1st ed.). Rio de Janeiro: Letra Capital. 
CUENYA, B. (2016). La política urbana frente a la mercantilización y elitización de la ciudad: algunas reflexiones y referencias a la situación argentina. Cuaderno Urbano, 21(21), 1-17.

CuenyA, B., \& CorRal, M. (2011). Empresarialismo, economía del suelo y grandes proyectos urbanos: El modelo de Puerto Madero en Buenos Aires. Eure, 37(111), 25-45.

CuenYA, B., \& GonZÁlez, E. (2017). Planificación y redistribución de beneficios del desarrollo urbano: la contribución compensatoria en Rosario, Argentina. Revista INVI, 32(90), 77-104.

De MAtTos, C. (2007). Globalización, negocios inmobiliarios y transformación urbana. Nueva Sociedad, (212), 82-96.

-- (2016). Financiarización, valorización inmobiliaria del capital y mercantilización de la metamorfosis urbana. Sociologias, (42), 24-52.

díAz OruetA, F., \& LouRÉs SeoANe, M. L. (2013). Neoliberalismo, políticas urbanas y reconfiguración socioespacial. Quid 16, 3, 7-16.

GargantinI, D., \& PeREsinI, N. (2017). Representaciones y estrategias de articulación-acción de actores públicos y privados en relación al gobierno del suelo urbano en la ciudad de Córdoba (Argentina). Estudios Socioterritoriales. Revista de Geografía, No 21, 155-171.

GoIcocheA, M. E. (2014). La ciudad de Buenos Aires como ámbito y objeto de negocios. Reflexiones en torno a la gestión urbana del Distrito Tecnológico Parque Patricios. Quid, 16(4), 161-185.

GuevarA, T. (2015). ¿La ciudad para quién? Transformaciones territoriales, políticas urbanas y procesos de producción del hábitat en la Ciudad de Buenos Aires (1996-2011). D.F. México, PUEC/UNAM.

HARVEY, D. (1989). From managerialism to entrepreneurialism: the transformation in urban governance in late capitalism. Geografiska Annaler. Series B, Human Geography, 71(1), 3-17.

-- (2007a). Espacios del capital. Hacia una geografía crítica. Madrid, Akal.

-- (2007b). Neoliberalism as Creative Destruction. The ANNALS of the American Academy of Political and Social Science, 610(1), 21-44.

Holanda, B., Rosa, S. V., \& Pequeño, R. (2017). Las asociaciones público-privadas y el desarrollo urbano análisis de ilegalidades en la creación de operaciones urbanas consorciadas en fortaleza, Ceará-Brasil. Entretextos, Año 9, N²6-, 2-16.

INDOVINA, F.(COORD). (2007). La Ciudad de baja densidad: lógicas, gestión y contención. Barcelona, Diputació Provincial de Barcelona.

JANOSCHKA, M. (2002). El modelo de ciudad latinoamericana. Privatización y fragmentación del espacio urbano de Buenos Aires: el caso Nordelta. EURE (Santiago), 28(85), 80-117.

JARAmiLlo, S. (2009). Hacia una teoría de la renta del suelo urbano (2 edición). Bogotá, Colombia: Universidad de los Andes.

Lefebvre, H. (2013). La producción del espacio ( $1^{\circ}$ español). Madrid, Capitan Swing.

Lemma, M. (2017). Gestión municipal y planificación urbana en Córdoba, Argentina (1983-2011). Urbe. Revista Brasileira de Gestão Urbana, 9(3).

Marengo, C. et. al. (2006). La Periferia de Córdoba: cuestiones sobre hábitat urbano. (C. Marengo, Ed.). Córdoba: Departamento de publicaciones FAUD - UNC. 
PeCk, J., Theodore, N., \& Brenner, N. (2013). Neoliberal Urbanism Redux? International Journal of Urban and Regional Research, 37(3).

PíREZ, P. (2005). Expansión territorial, privatización y fragmentación en la configuracion metropolitana de Buenos Aires Cadernos. Cadernos Metrópole, 13(enero-junio), 11-46.

-- (2016). Las heterogéneas formas de producción y consumo de la urbanización latinoamericana. Quid 16. Revista Del Área de Estudios Urbanos, 0(6), 131-167.

REESE, E. (2003). Instrumentos de gestión urbana, fortalecimiento del rol del municipio y desarrollo con equidad. Carajillo de La Ciudad, 18.

SMOLKA, M. (2012). Una nueva mirada a la recuperación de plusvalías en América Latina. Land Lines, (G), HJh.

SocolofF, I. C. (2013). Reflexiones en torno a las relaciones entre empresa, estado y ciudad: Un estudio a partir del caso IRSA en Buenos Aires (1991-2012). Tesis Doctor en Ciencias Sociales, Facultad de Ciencias Sociales, Universidad de Buenos Aires. 2013.

Theodore, N., PeCk, J., \& BrenneR, N. (2009). Urbanismo neoliberal: la ciudad y el imperio de los mercados. Temas Sociales SUR, (66), 12.

TOPALOV, C. (1978). La urbanización capitalista. Algunos elementos para su análisis. México: Edicol.

VAINeR, C. (2004). Pátria, empresa e mercadoria. En A cidade do pensamento único. Desmanchando consensos. (2, pp. 1-192). Petrópolis RJ- Brasil: Ed. Vozes.

VICUÑA DEL Río, M. (2013). El marco regulatorio en el contexto de la gestión empresarialista y la mercantilización del desarrollo urbano del Gran Santiago, Chile. Revista INVI, 28(August 2013), 181-219.

\section{FUENTES ELECTRÓNICAS}

CIPUV, Centro de InVestigación de Política URBana y Vivienda (2015). Atlas de Crecimiento Urbano. Universidad Torcuato di Tella. Buenos Aires, Argentina. (Consulta: 10/05/2017).

CDC, Consejo deliberante de la ciudad de Córdoba. Ordenanza Convenios Urbanísticos Publicos y/o Privados, Pub. L. No. 6 de septiembre del 2012, 1 (2012). Córdoba, Argentina: Digesto municipal. (Consulta: 20/02/2018).

DUARTE, J., \& BAER, L. (2013). Recuperación de plusvalías a través de la contribución por mejoras en Trenque Lauquen, Provincia de Buenos Aires-Argentina. In Foro Latinoamericano sobre instrumentos notables de intervención urbana (pp. 1-31). Recuperado de https://www.lincolninst.edu/sites/default/files/pubfiles/duartewp14jd1sp-full 0.pdf. (Consulta: 26/09/2017).

INDEC, Instituto nacional de estadísticas y censos (2010). Censo Nacional de Población, Hogares y Viviendas. (Consulta: 20/02/2018)

MARICATO, E. (2010). O Estatuto da cidade periférica. Aliança de Cidades e Ministério das Cidades (orgs.) O Estatuto da Cidade comentado. Recuperado de http://www.citiesalliance.org/sites/citiesalliance.org/files/CA Images/CityStatuteofBrazil Spanish Ch1.pdf.

(Consulta: 20/02/2018)

MARICATO, E., \& FERREIRA, J. (2002). Operação urbana consorciada: diversificação urbanística participativa ou aprofundamento da desigualdade?. Estatuto da Cidade e Reforma Urbana: novas perspectivas para as cidades brasileiras. (pp. 1-18). Porto Alegre: Sergio Antonio Fabris. Recuperado de http://www.fau.usp.br/cursos/graduacao/arg urbanismo/disciplinas/aup0268/20. Semestre 2012/Maricato whit aker operacoesurbanasconsorc.pdf. (Consulta: 20/02/2018) 
PBA, Provincia de Buenos Aires (2012). LEY 14449, Pub. L. No. 14449. Retrieved from http://www.gob.gba.gov.ar/legislacion/legislacion/l-14449.html. (Consulta: 20/02/2018).

PM, Provincia de Mendoza (2009). Ley de ordenamiento territorial de Mendoza, Pub. L. No. 8051.. Retrieved from http://www.mendoza.gov.ar/wp-content/uploads/sites/14/2014/09/Ley-Ordenamiento-TerritorialMendoza.pdf (Consulta: 20/02/2018). 